\title{
EL POTENCIAL TRANSFORMADOR DEL JUEGO EMERSIVO DESDE EL PUNTO DE VISTA DE LAS PRÁCTICAS ARTÍSTICAS
}

\author{
Samuel Gallastegui González \\ Universidad del País Vasco/Euskal Herriko Unibertsitatea, Dpto. Arte y Tecnología. Doctor
}

\section{Resumen}

Este artículo trata del potencial transformador que surge en los juegos digitales al realizar un cambio de orientación en el vector de atención, que de fuera hacia dentro, o inmersivo, pasa a ser de dentro hacia fuera, o emersivo. Por consiguiente, como los juegos dejan de ser estructuras ensimismadas, se reconoce y explota su capacidad de influir en la persona y la sociedad. Asimismo, se vincula la emersión de los juegos digitales con la emersión de ciertas prácticas artísticas que tratan de influir explícitamente en el individuo y la sociedad para provocar cambios tangibles. En este sentido, el artículo investiga las tecnologías blandas generadas por el juego emersivo y se pregunta qué tienen éstas que ofrecer al campo del arte. Esta investigación considera la emersión de los juegos digitales como un posible referente de tecnologías blandas que pueden ser aplicadas en las prácticas artísticas. En primer lugar examina cómo han sido conceptualizados los límites entre los juegos digitales y la vida cotidiana, en segunda instancia, se exploran posibles maneras de transgredir dichos límites, y, por último, se reconoce el potencial transformador del juego emersivo.

Palabras clave: PRÁCTICAS ARTÍSTICAS; JUEGOS DIGITALES; EMERSIÓN; TECNOLOGÍAS BLANDAS

\section{THE TRANSFORMATIVE POTENTIAL OF EMERSIVE GAMEPLAY FROM THE POINT OF VIEW OF ART PRACTICES}

\section{Abstract}

This paper deals with the transformative potential that is brought forth in digital games when a change in the attention vector is made, which from being outside in, or immersive, turns to be inside out, or emersive. Thus, since games cease to be self-absorbed structures, their ability to influence the individual as well as society is recognized and exploited. Likewise, the emersion in digital games is linked in this research with the emergence of certain artistic practices that explicitly seek to influence the individual and society to bring about tangible changes. In this sense, the paper researches the soft technologies that come about in emersive gameplay and inquires what have these to offer to the field of art. This research considers emersion of digital games as a possible reference of soft technologies that can be applied in artistic practices. First, it examines how the boundaries between digital games and everyday life have been conceptualized; second, possible ways to transgress those limits are explored; and third, the transformative potential of emersive gameplay is recognized.

Keywords: ART PRACTICES; DIGITAL GAMES; EMERSION; SOFT TECHNOLOGIES

\footnotetext{
Gallastegui González, Samuel. 2016. "El potencial transformador del juego emersivo desde el punto de vista de las prácticas artísticas". AusArt 4(2): 183-193 DOI: 10.1387/ausart.17168
}

\section{AUSART}




\section{PROBLEMA DE INVESTIGACIÓN}

El arte ha sufrido procesos de emersión en ciertos momentos de la historia, caracterizados por un cambio de orientación en los vectores de atención que, de ser desde fuera hacia dentro del arte, pasan a ser desde dentro hacia fuera; saliendo de su propio campo, sus estructuras, sus medios o sus espacios y derribando así las fronteras entre arte y vida. Por ejemplo, en los años 60 aconteció una emersión del arte hacia la vida. Durante la primera mitad del siglo XX las prácticas artísticas se habían caracterizado por el ensimismamiento y la inmersión. Encontramos numerosos ejemplos, desde los collages de Picasso hasta el ready-made de Duchamp, de obras de vanguardia que introducen objetos de la vida ordinaria en el territorio del arte. Pero en la segunda mitad del siglo parece que el fenómeno se invirtió, el arte comenzó a emerger, a tornarse desde dentro hacia fuera: salió de sus espacios -el museo y la galería- al espacio urbano y la naturaleza, como, por citar un caso, las obras de arte en la naturaleza de Richard Long; salió de sus medios y su especialización, combinando diferentes disciplinas, como, por ejemplo, las acciones sonoras de John Cage; y salió de su propio campo o mundo del arte, como las prácticas artísticas de acción política de Joseph Beuys.

Este cambio de orientación que torna el arte hacia la vida se produjo emborronando los límites entre ambos, como sintetizó Kaprow con su ya famosa cita "the line between the Happening and daily life should be kept as fluid, and perhaps indistinct, as possible" ([1993] 2003, 60). Kaprow considera este cambio de orientación una tendencia secularizante que siempre ha estado presente en el arte y que en los años 1950 y 1960 se convirtió en un impulso de arte-como-vida que pasó a dominar la vanguardia. Como resultado, la vida cotidiana, al ser empoderada por el arte, se cargaba de poder metafórico (224) expandiendo el campo de las prácticas artísticas.

El proceso de fundir el arte y la vida se produce muchas veces en forma de juego, como una actitud lúdica que desacraliza estructuras estables, actúa hacia la materialización de nuevas realidades posibles y reinterpreta la propia realidad. El juego devuelve el arte al mundo ordinario o secular, enfrentándose a ciertos principios estructurantes de nuestra sociedad, como por ejemplo, la separación entre lo serio y lo lúdico, o entre trabajo y juego; el problema parece estar, precisamente, en que la dicotomia juego-trabajo no está bien construida (Kaprow [1993] 2003, 116).

Las vanguardias artísticas tenían, efectivamente, una actitud lúdica que transformaba el arte, pero cuando es el propio juego el que emerge a la vastedad 
del mundo no-lúdico, tiene aún más poder transformador. Esta posibilidad de convertirlo todo en juego, de producir nuevos usos de las estructuras y posibilitar la creación de nuevas realidades, enlaza con ciertos planteamientos que se han realizado en el campo de la ludología. Castronova (2013), por ejemplo, plantea que el juego puede ser un campo de pruebas para cambios sociales y McGonigal (2011) que tiene el potencial para aplicar la inteligencia colectiva a la configuración de nuevas realidades. Tanto el juego como el arte construyen un contorno de separación con la realidad, creando un ámbito donde rigen unas condiciones particulares. Sin embargo, ambos tienen la capacidad de renegociar continuamente esta intersección entre sí mismos y la vida, redibujando líneas siempre fluidas en sus fronteras (Montola, Stenros \& Waern 2009).

Asimismo, el activismo artístico y la práctica social del arte pueden, de acuerdo con Flanagan (2010), transformarse mediante la acción lúdica. El poder que tiene el arte al tornarse hacia la vida es precisamente esa ambigüedad y pluralidad de roles, que lo hace susceptible de nuevas lecturas, de nuevas posibilidades. El poder de los juegos es crear dinámicas que tienen un efecto tangible en la realidad, dado que las acciones lúdicas pueden tener consecuencias en la realidad ordinaria.

La cuestión es que ciertos fenómenos lúdicos de los últimos años se han volcado hacia la creación de nuevas estructuras político-sociales (Castronova 2013), el coaprendizaje y la inteligencia colectiva (McGonigal 2011) o el activismo y la práctica social (Flanagan 2010), lo cual ha supuesto un cambio de orientación de dentro hacia fuera, una emersión a la realidad y la vida cotidiana, en contraposición al esquema de inmersión que se encuentra en los videojuegos más tradicionales, en los que la persona se sumerge -desde fuera hacia dentro- en la realidad propuesta por el juego. Además, este viraje hacia la realidad ordinaria se relaciona con el objetivo de ciertas prácticas artísticas de sacar el arte hacia la cotidianidad y transformarla, que en el caso de Fluxus o Guerrilla Girls, por citar dos ejemplos con repercusión, era explícito. Tanto en los casos lúdicos como artísticos, parece que el cambio de orientación hacia la realidad y vida cotidiana genera nuevas tecnologías blandas que tienen un gran potencial de transformación de las estructuras establecidas, luego parece conveniente hacerse la siguiente pregunta:

¿Qué tienen que ofrecer al arte las tecnologías blandas que se generan en la emersión (o cambio de orientación desde dentro hacia fuera) de los juegos digitales? 


\section{PROPÓSITO}

Este artículo propone la hipótesis de que el cambio de orientación de dentro hacia fuera en los juegos digitales ofrece nuevas oportunidades de aprender, empoderarse y contribuir a la sociedad, lo cual constituye un gran potencial transformador del que se pueden apropiar las prácticas artísticas.

Se establecen dos objetivos: el primero es describir las tecnologías blandas que acontecen en el juego emersivo; y el segundo mostrar estrategias lúdicas que puedan ser incorporadas a las prácticas artísticas para expandir su potencial transformador individual y colectivo.

Estos objetivos se alcanzarán mediante investigación bibliográfica, revisando diferentes fenómenos lúdicos en los cuales existe un emborronamiento de los límites entre vida y juego. Asimismo, se estudiarán los conceptos de "juego serio" y "ludificiación" como formas en las que los juegos emergen a la vida ordinaria e influyen en la sociedad. Finalmente, se analizará el potencial transformador de los juegos y se mostrará un ejemplo.

\section{EL "CÍRCULO MÁGICO"}

Igual que el cambio de orientación del arte hacia la vida parte de la negación del límite entre ambos (Kaprow [1993] 2003), en los juegos digitales esto sucede negando los límites del propio juego, que suelen ser referidos mediante el concepto de "círculo mágico", y reivindicando su capacidad de influir en las personas y la sociedad.

El "círculo mágico" es una metáfora propuesta por Huizinga (1938) para referirse al acuerdo por el cual ciertas personas, lugares y actividades se interpretan como pertenecientes al juego. Puede ser entendido como una barrera de contención para que lo lúdico no se desparrame en la realidad. Según Montola "The metaphorical magic circle of play is a voluntary, contractual structure that is limited in time and space" $(2005,16)$.

Si inmersión es una entrada de la persona en el "círculo mágico", cruzando esa barrera contractual que limita las acciones del juego, emersión es lo contrario, que los elementos que pertenecen al acuerdo establecido salgan a la realidad 
cotidiana. El concepto de "pervasividad" hace referencia a la capacidad de una estructura de extenderse más allá de sí misma. Aplicado a los juegos, se refiere a que el fenómeno lúdico emerge fuera del "círculo mágico", expandiendo los límites de éste. Montola junto con Stenros y Waern proponen que "a pervasive game is a game that has one or more salient features that expand the contractual magic circle of play spatially, temporally, or socially" $(2009,12)$.

La idea de que el juego esté filtrándose al dominio de lo ordinario constituye un nuevo paradigma en la relación con el jugador y con el mundo ordinario. En un juego pervasivo las acciones del juego pueden resultar inseparables de las acciones fuera del juego, ya que éste puede ser jugado en cualquier lugar, en cualquier momento y por cualquier persona (Montola, Stenros \& Waern 2009, 27). Esto crea la experiencia de que puede irrumpir en la realidad ordinaria en cualquier momento y cambiarla, ya que ésta ya no está tan a salvo como cuando se establecía una clara separación entre el mundo del juego y la realidad no-lúdica.

Resulta especialmente valiosa esta estrategia consistente en extender el "círculo mágico", porque así cualquier juego puede hacerse pervasivo, basta tan sólo con expandir sus fronteras temporales, espaciales y sociales, haciendo, por ejemplo, que un juego pueda ser jugado por todo el globo, o posibilitando que nuevas personas puedan agregarse durante el curso de la partida.

\section{JUEGOS DE REALIDAD ALTERNATIVA}

Los juegos de realidad alternativa surgen como una narración interactiva en red que utiliza diferentes medios para desarrollar una historia que se va modificando según la participación y las respuestas de los jugadores. Los medios principales para su desarrollo son Internet y sus recursos, tales como el correo electrónico, las páginas web, los mapas, las redes sociales y las aplicaciones en línea, entre otros.

El desarrollo de un juego de realidad alternativa combina la narrativa con la solución de problemas y conflictos para conseguir objetivos dentro del juego. Uno de los mayores objetivos de estos juegos es desdibujar las líneas entre el 
mundo del juego y el mundo real, o como explica Szulborski, negar su propia naturaleza de juego:

One of the main goals of an ARG is to deny and disguise the fact that it is even a game at all. This is what the community of immersive game fans and creators embrace as the main principle of Alternate Reality Games and what has come to be call the TINAG philosophy, for This Is Not A Game.

(Szulborski 2005, 1)

Las claves de estos juegos reside tanto en la colaboración participativa de muchos individuos, como en la ambigüedad sobre lo que pertenece al juego y lo que no, esto es, en la negación de su propia naturaleza lúdica. El potencial de este tipo de juegos se maximiza mediante la investigación de técnicas aprendizaje cooperativo aplicadas a la resolución de problemas (Bonsignore 2012, 252). El hecho de que estos juegos faciliten la organización emergente de sus jugadores y puedan funcionar como laboratorios de ideas los convierte en una herramienta poderosa para intervenir en los procesos de la realidad: se pueden utilizar en la educación, en el marketing, o en la investigación, por citar algunos ejemplos. Esto enlaza con el concepto de juegos serios, que son aquellos que tienen un objetivo más allá de lo lúdico.

\section{JUEGOS SERIOS}

El primer uso del oxímoron con un sentido muy semejante al actual lo utilizó Clark Abt en un su libro Serious Games (1970), como aquellos que "have an explicit and carefully thought-out educational purpose and are not intended to be played primarily for amusement. This does not mean that serious games are not, or should not be, entertaining" (Abt 1970, 9).

Los juegos serios proponen entornos virtuales y mecánicas de juego inmersivas, pero con un objetivo extralúdico o perteneciente al mundo ordinario. El hecho de que el propósito del juego esté relacionado con la realidad del jugador hace que éste se oriente hacia la realidad cotidiana. Por ejemplo, el videojuego PeaceMaker simula el conflicto israelí-palestino, utilizando imágenes del mundo real para plantear los enfrentamientos políticos, militares y sociales basados en esta realidad. 
Los jugadores tienen que reaccionar y tomar decisiones con el objetivo de solucionar el conflicto entre los dos Estados. El objetivo del juego, que otorga la condición de victoria, es la solución del conflicto y eliminación de la violencia, para lo cual el jugador tiene que tomar iniciativas e ir resolviendo los problemas que se le presentan como consecuencia de esas decisiones. La interfaz consta de un mapa de Israel, Cisjordania, y la franja de Gaza, un panel de decisiones y los indicadores económicos, sociales y políticos que informan del progreso del juego. Los eventos son representados de forma muy fidedigna, utilizando imágenes de medios periodísticos.

En este ejemplo, no se produce una inmersión en una realidad lúdica separada de la realidad ordinaria de la persona que juega, sino que, aunque se trate de una simulación, el juego centra la atención en un fenómeno fuera de lo propiamente lúdico, lo que supone para el jugador una toma de conciencia de un problema real. El propósito extralúdico además de tener un efecto transformador sobre la persona, tiene el potencial de influir y ayudar a resolver problemas reales.

Además de los juegos con un objetivo concienciador o pedagógico, existen juegos que facilitan investigaciones crowdsourcing, como Eterna, que consiste en diseñar moléculas y utiliza la creatividad de muchas personas para crear nuevos diseños de ácido ribonucleico. Del mismo estilo es Foldit, un rompecabezas en línea sobre las estructuras de la proteína, que forma parte de parte de una investigación, de manera que los investigadores analizan sólo las respuestas con una puntuación más alta para investigar su viabilidad.

Este tipo de juegos hacen que una actividad ardua se convierta en algo divertido, agradable e inmediatamente recompensante, lo cual enlaza con los el concepto de ludificación, que se refiere a la aplicación de mecánicas lúdicas a actividades que, en principio, no lo son.

\section{LUDIFICACIÓN}

Este potencial transformador que se ha descrito en los juegos serios entronca con el concepto de la ludificación o gamificación, adaptaciones del neologismo inglés gamification. Si juego serio es la creación de un juego para un uso que no sea solamente entretener, un proceso que lleva la vida al juego, ludificación 
hace referencia a la aplicación de mecánicas de juego a ámbitos no lúdicos, un proceso que lleva el juego a la vida.

Las estructuras ludificadas utilizan elementos lúdicos pero no dan lugar a juegos completos. El riesgo que tiene la ludificación es caer en la construcción de artefactos lúdicos y olvidarse de que el juego ha de construirse y pactarse socialmente (Deterding et al. 2011, 14). Por esta razón McGonigal (2011) critica duramente la gamificación. Ella contrapone el diseño "gameful" a la ludificación. Mientras la última se centra en los puntos, los niveles, los rankings y las insignias, el diseño "gameful" se centra en las emociones positivas, la relaciones, el sentido, los logros y la satisfacción personal. Es decir, se centra en la recompensa intrínseca del juego, al revés que las actividades ludificadas, que proponen una recompensa extrínseca al proceso.

El juego genera eustrés, estrés positivo, mientras que las emociones negativas generan distrés, estrés negativo. El diseño "gameful" se centra en el optimismo, crear objetivos voluntarios y provocar curiosidad, dando a los jugadores una habilidad concreta que puedan utilizar en la vida real. Así se incita a los jugadores a dar servicio a los demás, colaborando como fuerzas conjuntas para dar solución a problemas concretos.

\section{EL POTENCIAL TRANSFORMADOR DEL JUEGO EMERSIVO}

Csíkszentmihályi plantea la siguiente paradajoja: "How is it possible for play to be both divorced from reality and yet so rife with real life consequences?" $(1980,14)$. La separación entre juego y vida es una construcción que hemos realizado para contener los fenómenos lúdicos dentro de unos límites asumibles. Sin embargo, los juegos tienen un gran potencial transformador que se revela cuánto menos desvinculado está de la vida cotidiana.

Las acciones del juego, ligadas a las acciones de la vida ordinaria, aumentan el grado de repercusión que un juego tiene en la realidad. El juego, en tanto que virtual, parecería estar aislado del mundo, incapaz de generar ninguna consecuencia real. Y sin embargo no es así, los juegos tienen la capacidad real de generar cambios: en los principios y valores (p.e. McDonald's Video- 
game), en la realidad (p.e. Conspiracy for Good), o en la constitución física de la persona (p.e. Wii Fit), que resultan efectivos.

Castronova (2013) cree que los juegos digitales van a traer enormes beneficios a la sociedad. Según él, los juegos pueden ser un campo de pruebas para introducir pequeños cambios sociales. Si antes era una propiedad emergente del comportamiento humano que después se establecía, ahora el juego surge como estructura. El diseñador manipula la conducta del jugador en el juego, lo que hace que tenga mayor potencial de influencia en la sociedad, sobre todo cuando éste puede crecer exponencialmente en red.

Él se pregunta qué ocurre cuando el juego adquiere dimensiones tan grandes que lo hacen indistinguible de la realidad. Internet posibilita que cualquier juego sea jugado a escala global y que la reflexiones, las prácticas, las estrategias o las innovaciones sociales que se producen dentro de él puedan convertirse en el nuevo paradigma (Castronova 2013, 18).

Veamos, para finalizar, un caso que ilustra este potencial transformador así como la difuminación de los límites del "círculo mágico". World Without Oil es un juego que pide a sus jugadores que imaginen el mundo como si estuviera ocurriendo una crisis real del petróleo. Estuvo activo del 30 de abril de 2007 hasta junio de 2009, tiempo durante el cual, según la revista Current.org (2009), los jugadores ofrecieron formas de vivir con menos petróleo, enviaron consejos, videos instructivos e imágenes. En World Without Oil la imaginación colectiva de los participantes dio forma y soluciones a algo que podría pasar. Aunque está planteado de una forma hipotética, el juego ya no está orientado a un mundo lúdico, sino a un mundo potencial como el nuestro. Las soluciones que en él se dan sirven, sobre todo, para el mundo ordinario, no para un mundo virtual. No se produce una inmersión en el juego, sino un compromiso con un problema de la realidad ordinaria, que se trata de solucionar de forma lúdica.

Éste comenzaba con un simple "pongamos que el petróleo se ha acabado" y lo demás surgía sólo: hay que generar alternativas, aprender a vivir con ello. Los participantes realizaron todo tipo de artefactos y propusieron ideas que no sólo tenían sentido dentro del entorno lúdico limitado por ese "pongamos que...", sino que también eran aplicables a otras situaciones de la vida. Eran un auténtico "campo de pruebas" como dice Castronova $(2013,21)$. 
Juegos emersivos como World Without Oil podrían consistir, en sí mismos, en una práctica artística que utiliza tecnologías blandas con el objetivo de dar cauce a discursos alternativos y aumentar nuestra capacidad de transformarnos individual y colectivamente. El arte se podría apropiar de este gran potencial transformador simplemente incorporando a las prácticas artísticas estrategias como la creación de redes de inteligencia colectiva que comparten un objetivo lúdico, el uso de juegos con objetivos más allá de lo lúdico y la aplicación de mecánicas lúdicas.

\section{CONCLUSIONES}

El potencial transformador de los juegos parte de la eliminación de las barreras que limitan el juego a un estado ficticio o exclusivamente lúdico. Al reconocer la capacidad de afectar a las personas y los procesos sociales que tienen los juegos, adquieren un alcance mayor, produciendo efectos y consecuencias comprobables. Los juegos que aprovechan el potencial transformador trascienden las fronteras de lo lúdico e influyen en la sociedad.

El hecho de que el contenido del juego se oriente hacia el espacio físico y la vida hace que su diseño influya en estructuras que están más allá del mismo, como por ejemplo el trabajo, en las cuales tiene poder "secularizante" (Kaprow [1993] 2003), genera un gran impacto en la realidad ordinaria. Esto puede alcanzarse, por ejemplo, mediante el desarrollo de juegos que se convierten en un campo de pruebas y experimentación de pequeños cambios que podrían introducirse en la sociedad. En palabras de Castronova, "los juegos pueden verse como incubadoras de importantes cambios culturales" (2013, 21).

No se trata de que los juegos tengan influencia en la realidad, sino que se conviertan en la nueva realidad. Las prácticas artísticas pueden apropiarse de esta cualidad de los juegos para crear formas de arte colaborativas, ya que ofrecen la oportunidad de conectar las inteligencias individuales de una forma constructiva, haciendo, por ejemplo, que un trabajo arduo se convierta en algo divertido.

El cambio de orientación desde dentro hacia fuera de los juegos supone, en definitiva, un gran potencial de transformación que se manifiesta en la creación 
de diferentes estrategias para emerger a otras estructuras sociales, educativas, productivas o académicas. Un asunto que podría ser tratado por futuras investigaciones es, precisamente, explorar en la praxis artística las maneras en las que el arte puede apropiarse de las tecnologías blandas que surgen en este proceso de emersión.

\section{Referencias}

Abt, Crark C. 1970. Serious games. New York: Viking Press

Bonsignore, Elizabeth, Kari Kraus, June Ahn, Amanda Visconti, Ann Fraistat \& Allison Druin. 2012. "Alternate reality games: Platforms for collaborative learning". En The future of learning: Proceedings of the 10th International Conference of the Learning Sciences ICLS 2012, 251-8. Sydney: ICLS

Castronova, Edward. 2013. "Juegos e internet: un terreno fértil para el cambio cultural". En Cambio: 19 ensayos fundamentales sobre cómo Internet está cambiando nuestras vidas. Traducciones, Sebastián Durán, Mar Ozores \& Laura Vidal. Madrid: BBVA

Csikszentmihalyi, Mihalyi.1980. "Some paradoxes in the definition of play". En Play as Context, Alyce Taylor Cheska ed., 14-25. West Point, NY: Leisure Press

Deterding, Sebastian, Rilla Khaled, Lennart E. Nacke \& Dan Dixon. 2011 "Gamification: Toward a definition". En CHI 2011 Conference Proceedings and Extended Abstracts: The 29th annual CHI Conference on Human Factors in Computing Systems, Vancouver, BC, May 7-12, 1-4. New York: Association for Computing Machinery

Flanagan, Mary. 2010. "Creating Critical Play". En Artists rethinking games, edited by Ruth Catlow, Marc Garret \& Corrado Morgana, 49-53. Liverpool: Foundation for Art and Creative Technology

Huizinga, Johan. (1938) 2010. Homo ludens. Traducción de Eugenio Imaz. Madrid: Alianza Emecé

Kaprow, Allan. (1993) 2003. Essays on the blurring of Art and Life. Edited by Jeff Kelley. Berkerley CA: University of California

McGonigal, Jane. 2011. Reality is broken: Why games make us better and how they can change the world. NewYork: Penguin

Montola, Markus. 2005. "Exploring the edge of the magic circle: Defining pervasive games". En Design Automation Conference: Proceedings of the 42nd Design Automation Conference, Anaheim Convention Center, Anaheim, CA, June 13-17, 16-19. New York: Association for Computing Machinery

Montola, Markus, Jaakko Stenros \& Annika Waern. 2009. Pervasive Games: Theory and Design. Burlington, MA: Morgan Kaufman

Szulborski, Dave. 2005. This is not a game: A guide to alternate Reality gaming. Morrisville, NC: Lulu.com

(Artículo recibido 05-10-16; aceptado 20-12-16) 